\title{
Practical issues related to uterine pathology: endometrial stromal tumors
}

\author{
Marisa R Nucci \\ Division of Women's and Perinatal Pathology, Department of Pathology, Brigham and Women's Hospital, \\ Boston, MA, USA
}

\begin{abstract}
Uterine mesenchymal tumors continue to be a challenge to diagnose due to their non-specific clinical presentation, often non-distinctive gross appearance, varied (and many times overlapping) morphologic appearance, and unsuspected pitfalls in immunohistochemical expression. This review will focus on endometrial stromal tumors and those features that help in their distinction. In particular, a practical approach to the diagnosis of endometrial stromal neoplasia will be covered including recognition as a stromal process in a biopsy/curettage and distinction from a highly cellular leiomyoma. In addition, distinction of a stromal nodule from a low-grade endometrial stromal sarcoma (LGESS) and stromal sarcoma with limited infiltration in a hysterectomy specimen will be covered. The salient features that help distinguish a LGESS from a uterine tumor resembling ovarian sex-cord tumor as well as high-grade endometrial stromal sarcoma, the latter a tumor recently reintroduced in the WHO classification will also be discussed. Finally, a practical approach to the diagnosis of undifferentiated uterine sarcoma (UUS) will be presented. Modern Pathology (2016) 29, S92-S103; doi:10.1038/modpathol.2015.140
\end{abstract}

Endometrial stromal tumors can be divided into four main categories, which are currently recognized by the WHO $(2014)^{1-3}$ (A) endometrial stromal nodule (ESN), (B) endometrial stromal sarcoma, low-grade, (C) endometrial stromal sarcoma, high-grade, and (D) UUS. The purpose of this review is to highlight the salient clinical, morphologic, immunohistochemical, and genetic differences that allow their distinction.

\section{Endometrial Stromal Nodule}

ESNs are defined as tumors with absent to at most minimal myometrial invasion $(\leq 3 \mathrm{~mm}$ and $<3$ protrusions) and no vascular invasion. ${ }^{4,5}$ They occur much more commonly in the corpus than in the cervix. They are frequently polypoid with protrusion into the uterine cavity but may be intra-myometrial (ranging up to $10 \mathrm{~cm}$ ). They typically are well circumscribed and show a uniform tan to yellow, soft cut surface (Figure 1a). Cyst formation as well as ischemic-type necrosis and hemorrhage may be seen. They are characterized microscopically by a

Correspondence: Dr MR Nucci, MD, Division of Women's and Perinatal Pathology, Department of Pathology, Brigham and Women's Hospital, 75 Francis Street, Boston, MA 02115, USA. E-mail: mnucci@partners.org

Received 5 November 2015; accepted 5 November 2015 well-circumscribed, pushing border (Figure 1b); however, small irregularities present as lobulated or finger-like projections $(<3$ in number and $<3 \mathrm{~mm}$ ) into adjacent myometrium may occur. The neoplastic cells of ESNs resemble proliferative phase endometrial stroma, being composed of cells with uniform round to ovoid nuclei that have scant to moderate amounts of amphophilic to eosinophilic cytoplasm. These cells appear to whorl around the prominent vascular component, which resembles the spiral arterioles of non-neoplastic endometrium. The vessels are typically evenly spaced and uniform in caliber throughout the neoplasm; however, occasionally large, thick-walled vessels may be present, although this is usually only a focal finding identified in only a minority of cases. ESNs can show variant morphology, most commonly smooth muscle differentiation, ${ }^{6}$ but unusual forms of differentiation including skeletal muscle may occur. ${ }^{7}$ Immunohistochemistry for vimentin, CD 10, and actins as well as WT1, ER, and PR are typically positive (rarely tumors may be CD 10 negative). ${ }^{8}$ Desmin and h-caldesmon are positive in areas of smooth muscle differentiation although occasionally areas of conventional stromal differentiation may be desmin positive, and if so, typically it shows a perinuclear cytoplasmic pattern of staining. The most common genetic aberration identified in ESNs, which is similarly identified in LGESS (discussed later), is $\mathrm{t}(7 ; 17)(\mathrm{p} 15 ; \mathrm{q} 21) \cdot{ }^{9-12}$ 

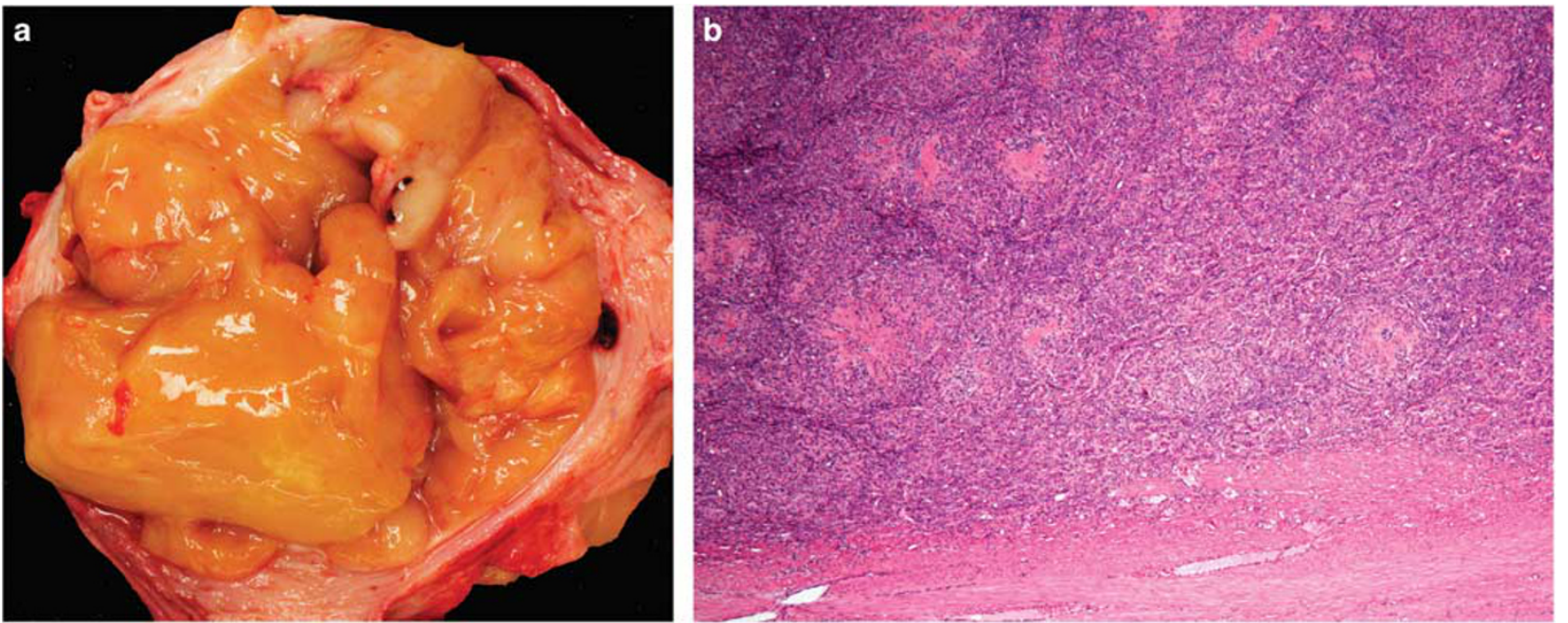

Figure 1 Endometrial stromal nodule. The tumor is characteristically well circumscribed with a tan to yellow cut surface; occasionally, it can show cystic degeneration as seen here (a). A well-delineated border with the myometrium is present (b).

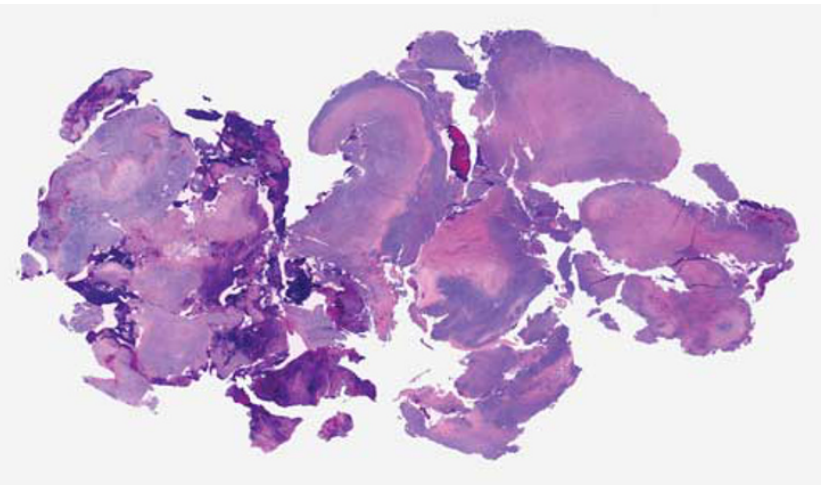

Figure 2 Endometrial stromal neoplasm (curettage specimen). Helpful features in recognizing a neoplastic stromal process in this setting include noticing multiple fragments of tissue composed entirely or almost entirely of endometrial type stroma with an expansile appearance.

\section{Recognition as an Endometrial Stromal Neoplasm (Biopsy/Curettage Specimen)}

The first clue that a biopsy/curettage specimen harbors an endometrial stromal neoplasm is the finding of multiple fragments of tissue composed entirely or almost entirely of endometrial-type stroma (Figure 2); in one study, most endometrial stromal tumors presented with fragments measuring $>5 \mathrm{~mm} .{ }^{13}$ In general, the stroma also has an expansive, sheet-like growth such that glands, if present, appear at the periphery, when there is involvement of the endometrium. The main pitfalls are distinction from aglandular functionalis (strips of endometrial stroma devoid of glands as a consequence of attenuation secondary to an underlying submucosal leiomyoma) in a reproductive woman and atrophic endometrial stroma lacking glands in a peri- or postmenopausal woman. In both instances, the total amount of endometrial stroma on the slide is less, resulting in smaller fragment size. In addition, the stroma is less robust or expansile in appearance. In a postmenopausal patient, an endometrial polyp with dense cellular stroma may also fall into the differential diagnosis as the glands are often atrophic and inconspicuous, which results in the stroma being more conspicuous at low power examination; however, careful scrutiny at high power will reveal the presence of uniformly placed benign glands.

\section{Distinction from Low-Grade Endometrial Stromal Sarcoma (Biopsy/Curettage Specimen)}

In the majority of cases, the distinction between an ESN and low-grade endometrial stromal sarcoma (LGESS) cannot be made with certainty in a curettage/ biopsy specimen as the margin of the tumor usually cannot be assessed well or completely. In these cases, the diagnosis of 'endometrial stromal neoplasia' should be rendered with a note stating that the distinction between a nodule and a sarcoma cannot be made. In some instances, bona fide lymphovascular invasion may be seen and the diagnosis of LGESS can be made, but this diagnosis should be made sparingly and only if the vascular invasion is convincing with exclusion of specimen artifact.

\section{Distinction from LGESS (Hysterectomy Specimen)}

ESN and LGESS have a similar clinical presentation (most commonly vaginal bleeding) and morphologic appearance. On gross examination, ESNs are well-circumscribed, non-encapsulated masses in contrast to most LGESSs, which typically show a multinodular growth within the endometrium and 

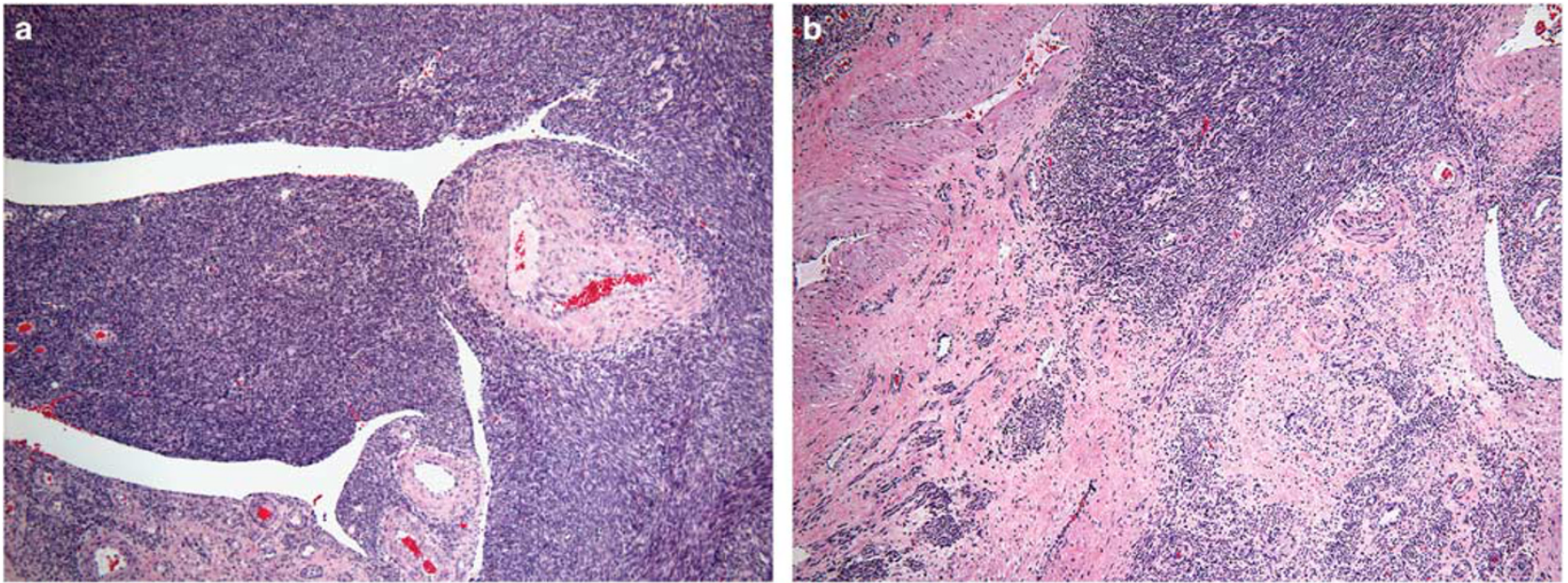

Figure 3 Highly cellular leiomyoma. Histologic features that favor a highly cellular leiomyoma include large, thick-walled blood vessels, cleft-like spaces, and fascicular growth (a). In addition, merging of the neoplastic cells at the periphery of the tumor with the myometrium, instead of infiltration, helps one avoid the pitfall of misinterpreting this benign tumor as a malignant stromal neoplasm (b).

the myometrium. Of note, LGESS may sometimes appear deceptively well circumscribed on gross examination and thus extensive sampling of the border of all tumors in which the diagnosis of an ESN is being entertained is imperative. ESNs are by definition well-circumscribed histologically, and this is the most important feature in distinguishing between stromal nodule and stromal sarcoma. Occasionally, there may be focal irregularities of the border of an ESN in the form of finger-like or nodular projections. These foci should not be greater than 3 in number and should not extend $>3 \mathrm{~mm}$ from the main tumor mass. In addition, vascular space invasion is exclusive of this diagnosis, and if present, the diagnosis of an LGESS should be rendered.

\section{Distinction from LGESS with Limited Infiltration (Hysterectomy Specimen)}

LGESS with limited infiltration represents an uncommon scenario, in which a tumor appears well circumscribed on gross examination mimicking a stromal nodule, and on histologic examination does not have the overt permeative growth of a LGESS, but also does not fulfill the histologic criteria for an ESN (ie, $<3$ finger-like projections $\leq 3 \mathrm{~mm}$ ). ${ }^{3}$ Of course, it is completely arbitrary where one draws the line between 'overt' permeation and permeation that breaches $3 \mathrm{~mm}$ from the main mass making this diagnosis even more challenging. A study examining 50 ESNs identified three such tumors, but only one with follow-up information. In that patient, the tumor had six permeative foci projecting from the main mass ranging in distance from 1 to $5 \mathrm{~mm}$, and the patient had no evidence of disease at 5 years; however, this follow-up is unfortunately too short to reach any firm conclusions. For practical purposes, one can diagnose these tumors as 'endometrial stromal sarcoma with limited infiltration' or 'endometrial stromal neoplasm with limited infiltration' with a note stating that the degree of infiltration is not as overt as typically seen in a stromal sarcoma and therefore, the tumor may pursue a benign clinical course; nevertheless, long-term clinical follow-up is recommended.

\section{Distinction from Highly Cellular Leiomyoma}

ESNs and highly cellular leiomyoma (HCL) can be easily confused as they have a similar clinical presentation (pain, bleeding, or may be an incidental finding) and gross appearance (yellow to tan, soft, and circumscribed). In addition, HCL is as cellular as a stromal neoplasm on histologic examination, leading to further potential confusion. However, the morphologic appearance of HCL differs in important aspects, which can be recognized in most cases by the following clues (Figure 3$)^{14}$ : (1) large, thick-walled vessels characteristically present throughout the tumor, which is in contrast to stromal tumors where large vessels are rare, and if present, tend to be located at the periphery of the nodule, (2) cleft-like spaces; a feature characteristic of a smooth muscle tumors in general, and (3) merging of the fascicles at the periphery with the myometrium, which may mimic infiltration; a feature that may be seen in conventional leiomyoma, but is more apparent when the tumor is cellular. Immunohistochemistry may be useful in distinguishing between these two tumors, but caution must be exercised to correlate the immunoprofile with morphology, as ESNs can contain areas of smooth muscle differentiation. Thus, the areas that have the morphologic appearance of endometrial stroma in an ESN with smooth muscle differentiation would typically be h-caldesmon negative whereas an HCL would be 
positive, albeit this marker may be less diffusely positive than in a conventional smooth muscle tumor. It should also be mentioned that HCL can show diffuse CD 10 positivity, and ESN can be desmin positive although usually the staining pattern is less diffuse, and tends to have a dot-like and/or peri-nuclear cytoplasmic pattern instead of the diffuse cytoplasmic positivity seen in a smooth muscle tumor.

\section{Practical take-home points}

(1) ESNs can sometimes show minimal and focal finger-like projections beyond its circumscribed border. Follow the ' 3 ' rule-the projections into the adjacent myometrium should not be $>3 \mathrm{~mm}$ from the main tumor mass and not $>3$ in number.

(2) LGESSs are occasionally deceptively well circumscribed on gross examination; thus, adequate sampling of the border/surrounding myometrium is imperative to reach the correct diagnosis. A good rule of thumb is one section per $\mathrm{cm}$; most sections should include the tumor-myometrial interface.

(3) Rarely, endometrial stromal tumors may show only limited infiltration into the myometrium. Therefore, it is important to state in the report that follow-up for such cases is limited. Of note, there should not be vascular invasion, as the presence of such warrants diagnosis as LGESS.

(4) In most instances, the distinction between an ESN and LGESS cannot be made in a biopsy/ curettage specimen and thus diagnosis as an 'endometrial stromal tumor', with a comment stating that the distinction between a nodule and a sarcoma cannot be made, is recommended. On occasion, lymphovascular invasion may be seen; this may be the one instance where a diagnosis of a stromal sarcoma can be made with certainty. Of note, both tumors share the same genetic aberration; thus, the presence of $t(7 ; 17)$ cannot be used to distinguish between the two entities.

(5) It is important to be aware that a highly cellular tumor composed of bland ovoid to spindled cells may represent either an endometrial stromal tumor or a HCL, and special attention to the histologic differences is key in reaching the correct diagnosis. If necessary, a panel of antibodies (CD 10, desmin, and h-caldesmon) should be employed as the use of any single marker may be misleading (eg, CD 10 is positive in smooth muscle tumors). Correlation of marker expression with the morphology will help distinguish a stromal nodule with smooth muscle differentiation from a pure smooth muscle tumor.

\section{Endometrial Stromal Sarcoma, Low Grade}

LGESS is the second most common pure mesenchymal malignancy of the uterus; and it affects women primarily in the peri-menopausal age group. ${ }^{15-18}$
Patients most commonly present with abnormal uterine bleeding or symptoms and signs related to extra-uterine spread (seen in approximately 1/3). On gross examination, the tumor much more commonly involves the uterine corpus than the cervix. It typically forms multiple poorly defined, often coalescent, tan to yellow, soft nodules within the endometrium and myometrium (Figure 4a). Areas that appear paler, firmer, and gray are typically seen in tumors with smooth muscle differentiation. Worm-like tumor plugs may be appreciated within vascular spaces and are usually more apparent in parauterine/parametrial soft tissues. Histologically, it characteristically demonstrates extensive permeation of the myometrium as irregular islands with frequent vascular invasion (Figure 4b). The majority of LGESSs show bland nuclear features with monotonous oval to spindle nuclei that resemble proliferative phase endometrial stroma (Figure 4c); mitotic activity is generally low ( $<5 / 10$ high power fields, but can be higher) and necrosis is usually absent. The stromal background contains a rich vascular network of small arterioles and capillaries, sometimes with hyalinized walls, and the tumor cells are frequently seen growing in a swirling pattern around these vessels. Collagen bands and plaques as well as histiocytes (either singly or in small groups) may be admixed with the tumor cells. Variant microscopic features that may be seen include: (1) smooth muscle differentiation (Figure 4d), (2) rhabdomyoblastic differentiation, (3) myxoid background, (4) fibroblastic appearance, (5) epithelioid differentiation, (6) sex cord-like differentiation, (7) endometrioid glands, (8) clear cells or granular cytoplasm, (9) rhabdoid features, (10) bizarre cells, (11) adipose tissue, and (12) papillary or pseudo-papillary growth. ${ }^{6,19-23}$

Immunohistochemically, the tumor cells are typically positive for CD10, vimentin, actins, WT-1, IFITM1, ER, and PR. ${ }^{8,24}$ Keratin may be positive in conventional areas, but also in areas of sex cord-like and glandular differentiation. ${ }^{25}$ Areas of sex-cord differentiation may also be positive for inhibin, calretinin, CD 99, and Melan-A. ${ }^{26}$ Desmin and h-caldesmon are usually positive in areas of smooth muscle differentiation; ${ }^{27,28}$ desmin may be occasionally positive in conventional areas or areas of sex-cord differentiation. Rarely, LGESS may contain cells with clear cytoplasm, which can be HMB45 positive. ${ }^{29}$

The most common genetic abnormality in low-grade ESS is $t(7 ; 17)(\mathrm{p} 15 ; \mathrm{q} 21)$, resulting in the fusion of JAZF1 and SUZ12(JJAZ1) genes at 7p15 and 17q21, respectively. ${ }^{9-12,30,31}$ The reported frequency of JAZF1-JJAZ1 fusion has been detected in 48\% of low-grade ESS. ${ }^{32}$ The second most frequent abnormality is $\mathrm{t}(6 ; 7)(\mathrm{p} 21 ; \mathrm{p} 15)$, a so-called variant translocation of the $t(7 ; 17)$, because of the involvement of $7 \mathrm{p} 15$ and $6 \mathrm{p} 21$ instead of $17 \mathrm{q} 21$ resulting in a JAZF1-PHF1 fusion gene. ${ }^{31,33}$ The PHF1 gene at 6p21 can also fuse with EPC1 at 10p11 and MEAF6 at 1p34. Of note, there appears to be a correlation in 

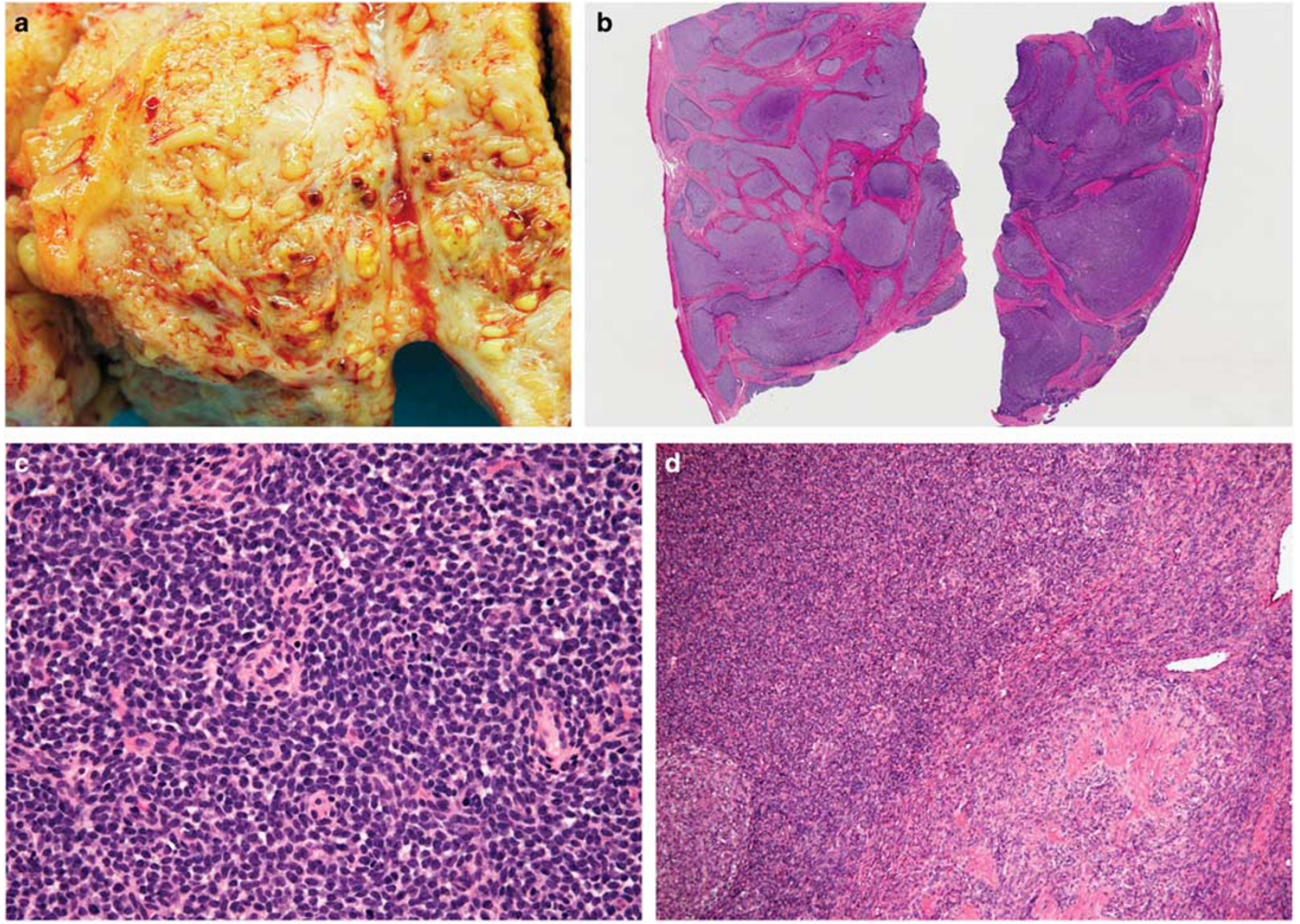

Figure 4 Endometrial stromal sarcoma, low grade. On gross examination, this tumor typically forms multiple tan to yellow soft nodules in the myometrium (a). These nodules correspond histologically to extensive permeative growth within the myometrium as well as lymphovascular involvement (b). At higher power magnification, the tumor cells typically resemble that of proliferative phase endometrial stroma (c). The most common variant microscopic feature that may be seen is smooth muscle differentiation, which in this image has a 'starburst' appearance. Note the merging with conventional stromal neoplasia in the upper left hand part of the image (d).

ESS showing sex cord-like differentiation having PHF1 genetic rearrangement. ${ }^{34}$ Two additional translocations have been described in ESSs, $t(X ; 22)$ $(\mathrm{p} 11 ; \mathrm{q} 13)$ and $\mathrm{t}(\mathrm{X} ; 17)(\mathrm{p} 11.2 ; \mathrm{q} 21.33)$ associated with a $Z C 3 H 7 B-B C O R$ fusion and a MBTD1-CXorf67 fusion, respectively. ${ }^{35,36}$ Although endometrial stromal tumors are genetically heterogeneous, the different genes involved in low-grade ESS are functionally related (PHF1, SUZ12, EPC1, MBTD1), being members of the polycomb gene family. Of interest, ZC3H7B-BCOR, MEAF6-PHF1, and EPC1-PHF1 fusions were also identified in ossifying fibromyxoid tumors and JAZ1-PFH1 in an ossifying sarcoma of the heart. Rarely, endometrial stromal tumors can show MDM2 amplification by FISH as well as MDM2 expression by immunohistochemistry, a potential pitfall particularly in tumors occurring in locations more common to liposarcoma (ie, peritoneum and retroperitoneum). ${ }^{37}$

Surgical stage appears to be the most important prognostic factor. ${ }^{38}$ Patients with low-grade ESS $^{32}$ have an excellent prognosis with a 90\% 5-year disease-free survival if low stage (I/II); 5-year survival drops to $50 \%$ if high stage (III/IV). ${ }^{39}$

\section{Distinction from Highly Cellular Intravenous Leiomyomatosis}

Both highly cellular intravenous leiomyomatosis (IVL) and LGESS are characterized by prominent intravascular growth, a tan to yellow cut surface, and frequent association with a dominant uterine mass (Figure 5a). ${ }^{40-42}$ Extra-uterine extension with 'worm-like' plugs of tumor within vascular spaces may be seen in both entities. A further confounding factor is the prominent cellularity seen in highly cellular IVL, which parallels that seen in stromal neoplasms (Figure 5b). Histologic features that favor highly cellular IVL include: (1) a clefted or lobulated contour of the intravascular component, often with hydropic change, (2) fascicular growth, (3) cells with nuclei that have blunted ends, and (4) more than occasional prominent thick-walled vessels 

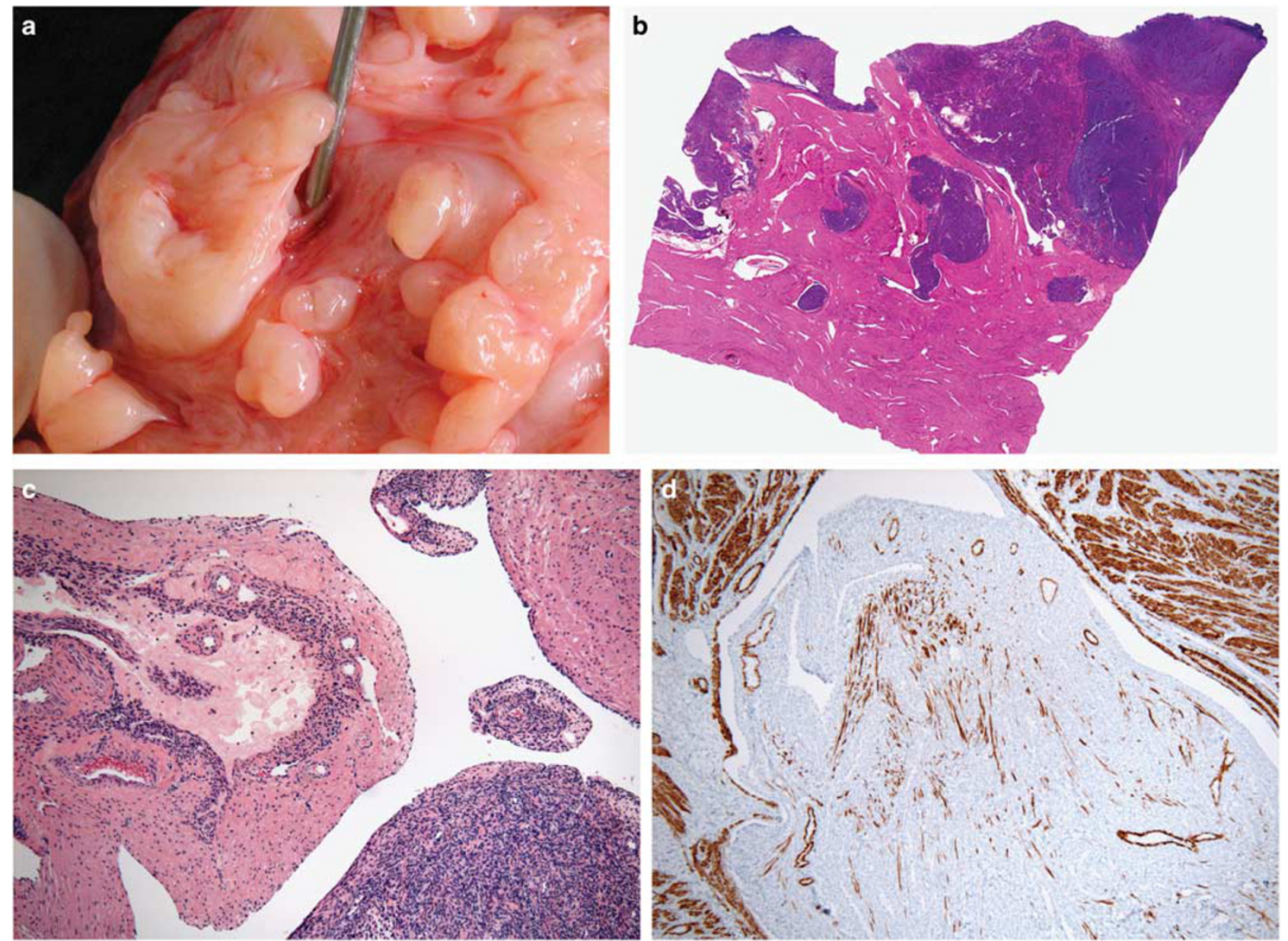

Figure 5 Highly cellular intravenous leiomyomatosis. This tumor can grossly mimic an endometrial stromal sarcoma as both can show prominent intravascular growth with a tan to yellow cut surface (a). The marked cellularity of the cellular smooth muscle component can also closely mimic stromal neoplasia on low power magnification (b); however, clues to the diagnosis include hydropic change as well as a clefted and lobulated contour of the intravascular component (c). The presence of h-caldesmon positivity, which may be focal, supports the diagnosis of intravenous leiomyomatosis (d).

(Figure 5c). A panel of immunohistochemical stains may be useful and should include CD10, h-caldesmon, and desmin. As occurs with HCL, highly cellular IVL may show diffuse CD10 positivity and decreased h-caldesmon expression; however, h-caldesmon is usually at least focally positive (Figure 5d). Correlation with morphology is key. For example, finding nodules of tumor expressing h-caldesmon that are morphologically identical to nodules that have lost expression still supports the entire tumor as representing a smooth muscle neoplasm.

\section{Distinction of LGESS with Sex Cord-Like Differentiation from a Uterine Tumor Resembling an Ovarian Sex-Cord Tumor}

Uterine tumor resembling ovarian sex-cord tumor (UTROSCT) is a rare uterine mesenchymal neoplasm that is composed entirely of epithelial-like cells with architectural patterns resembling those described in sex-cord stromal tumors of the ovary (Figure 6a). ${ }^{43,44}$ These tumors are usually well circumscribed, but can show infiltration of the myometrium and are often yellow. These features, along with the fact that endometrial stromal tumors can show extensive sex cord-like differentiation, can lead to diagnostic confusion. The distinction between LGESS and UTROSCT is based on the finding of areas of conventional stromal neoplasia in the former by H\&E examination, but this cannot be done in the majority of cases in a biopsy or curettage specimen. Immunoperoxidase stains may be helpful as UTROSCT should be positive for at least one sex-cord stromal marker whereas endometrial stromal tumors should be negative for these in the areas of conventional stromal neoplasia if present in the sample. A panel including inhibin, calretinin, and Melan A are the most useful (Figure 6b); CD99 and CD56 may also be positive ${ }^{44,45}$ The distinction between a UTROSCT and a LGESS is clinically relevant as most UTROSCT behave in a benign 

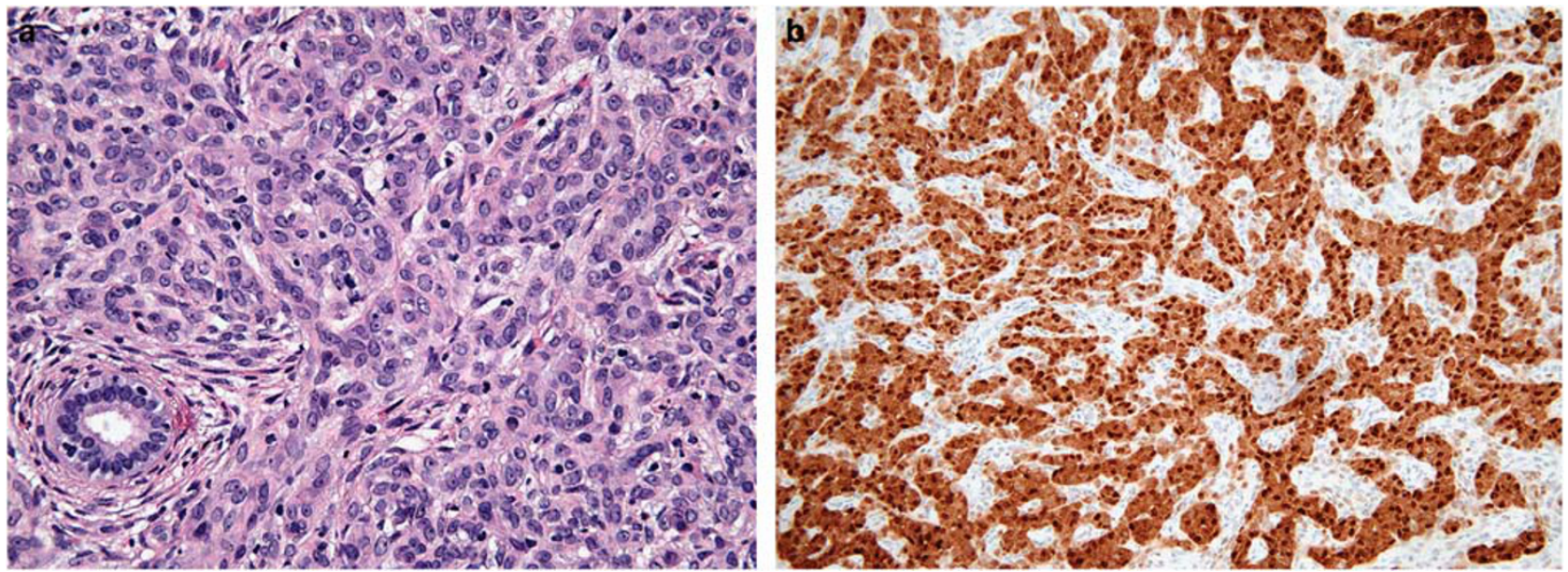

Figure 6 Uterine tumor resembling ovarian sex-cord tumor (UTROSCT). These unusual tumors are composed of epithelial-like cells that recapitulate the architecture patterns described in ovarian sex-cord stromal tumors (a). They are typically positive for at least one sex-cord stromal marker; in this case, calertinin was diffusely positive (b).

manner (rare cases have an aggressive clinical course), whereas patients with LGESS have an indolent, but protracted, clinical course with frequent recurrences.

\section{Practical take-home points}

(1) One should be aware of the variety of variant microscopic features that may be seen in LGESS in order to reach the correct diagnosis.

(2) IVL can be highly cellular and mimic a LGESS. Helpful histologic features include a clefted or lobulated contour of the intravascular component, fascicular growth, cells with nuclei that have blunted ends, and more than occasional prominent thick-walled vessels.

(3) Sex cord-like differentiation is the defining histologic feature of UTROSCT but can also occur, in varying amounts, in LGESS (as well as stromal nodules). As any component of stromal neoplasia will exclude UTROSCT, the tumor must be sampled extensively before this diagnosis is made. Thus, the diagnosis of UTROSCT should not be made definitively in a biopsy/curettage specimen.

\section{Endometrial Stromal Sarcoma, High Grade}

High-grade endometrial stromal sarcoma (HGESS) is a stromal neoplasm displaying uniform nuclear features that are intermediate between classic low-grade ESS and UUS. They have a characteristic genetic abnormality- $t(10 ; 17)(q 22 ; p 13)$ associated with a YWHAE-FAM22 (NUTM2AB) fusion-and are frequently associated with a morphologically low-grade fibromyxoid component. ${ }^{2,46-49}$ These tumors are uncommon; patients' ages range from the third to the seventh decade with a mean age of 50 years. They most commonly present with abnormal uterine bleeding and/or symptoms related to extra-uterine spread as tumors often are high stage (Figure 7a). They typically form a polypoid, intracavitary mass and often are poorly circumscribed with myometrial invasion; areas of hemorrhage and necrosis are also commonly present. Histologically, the tumor typically has an extensive permeative growth with extensive finger-like myometrial and vascular invasion; destructive myometrial infiltration is also common. The tumor often contains both morphologically low- and high-grade areas appreciable on low power examination as hyperand hypocellular areas (Figure 7b), but it is composed of a uniform population of tumor cells, even in the high-grade areas, with no apparent nuclear pleomorphism at low power magnification ( $\times 4-\times 10$ objective). The morphologically high-grade areas typically show nested and corded growth (Figure 7c). In contrast to LGESS, in these high-grade areas there is a prominent delicate arborizing capillary network in which small concentric arterioles are only present occasionally. The tumor cells in the high-grade areas are round, epithelioid cells with scant to moderate amounts of eosinophilic cytoplasm; they have round to oval vesicular nuclei with irregular nuclear contours and nucleoli. As a point of reference, the nuclei are typically 4-6 times the size of a lymphocyte. Mitotic activity is brisk, typically greater than 10 mitoses per 10 high power fields. The morphologically low-grade (hypocellular) areas often have a fibroblastic or myxoid appearance (Figure $7 \mathrm{~d}$ ), similar to that seen in the fibrous and myxoid variants of LGESS. They are composed of cells with round to oval nuclei and scant cytoplasm; and mitotic activity is usually much less apparent. It is important to be aware that the low- and high-grade components have a different immunohistochemical profile (Figures 7e and f). ${ }^{50}$ The low-grade component is typically positive for CD10, ER, and 


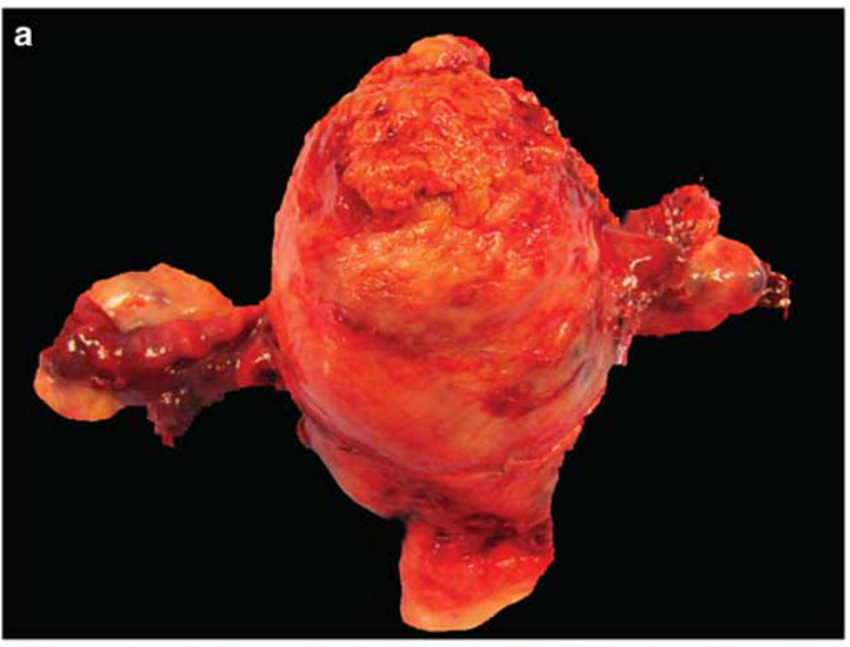

b
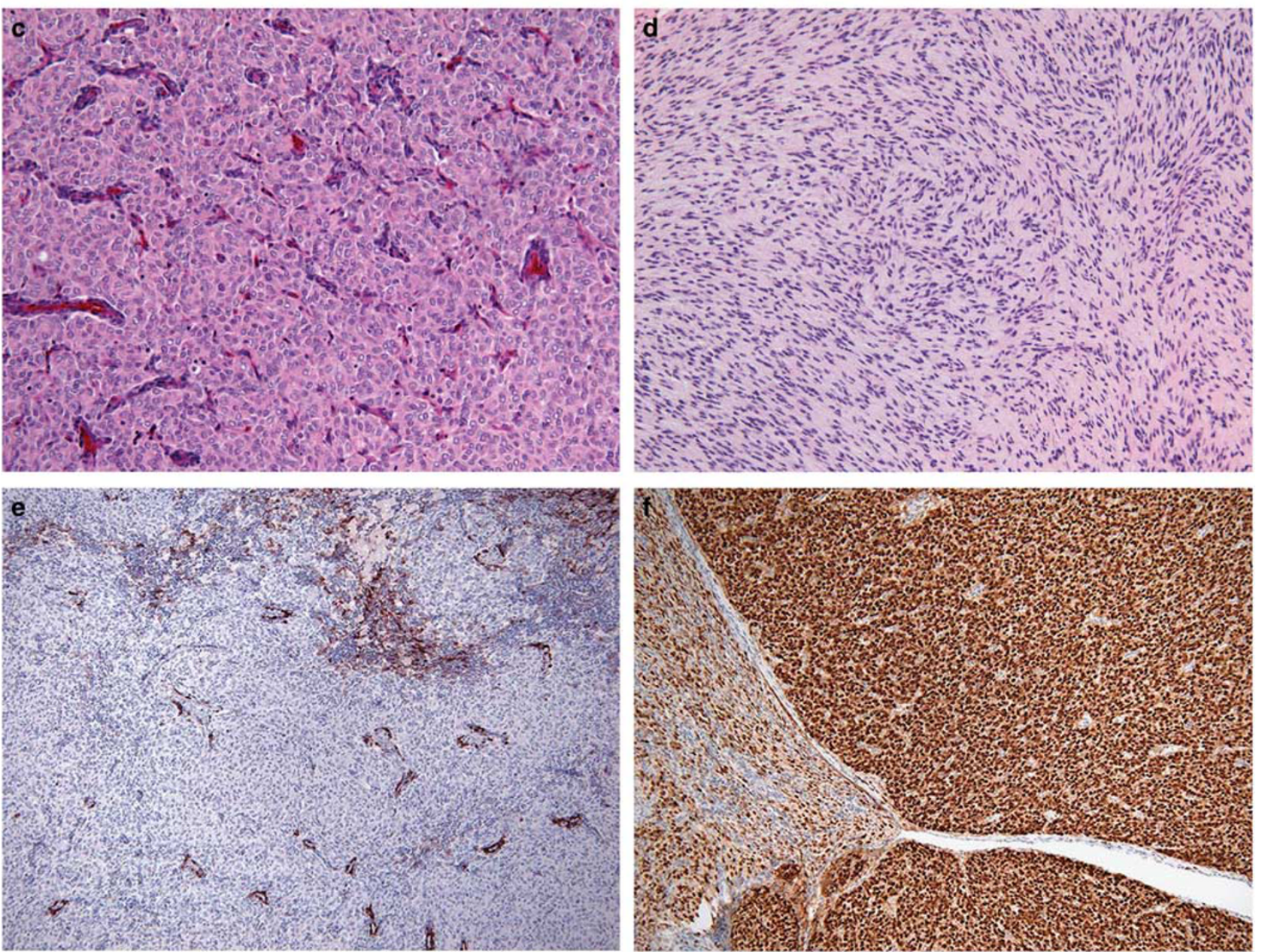

Figure 7 Endometrial stromal sarcoma, high grade. On gross examination, this tumor often presents with extension beyond the uterus (note serosal involvement) and is a clue to the diagnosis. (a) Histologically, the tumor extensively infiltrates the myometrium and typically shows a biphasic growth, which may be appreciable upon low power magnification (b). The high grade component typically shows a nested and corded growth and is composed of uniformly sized round cells with oval vesicular nuclei and moderate amounts of eosinophilic cytoplasm (c). The low-grade component shows morphologic similarity to the fibromyxoid variant of low-grade endometrial stromal sarcoma (d). The high-grade epithelioid areas are typically negative for CD10 whereas the low-grade areas are usually positive (e). Cyclin D1 is characteristically strongly and diffusely positive in the high-grade component and variably positive in the low-grade component (f). 
PR and will show patchy positivity (usually weak and focal) for cyclin D1; while CD117 is negative. In contrast, the high-grade component is typically negative for CD10, ER, and PR, and shows strong and diffuse positivity ( $>70 \%$ tumor cell nuclei) for cyclin D1; CD117 is often positive. ${ }^{51}$

Patient with high-grade ESS typically present with advanced stage disease (stages II-IV) and frequently have recurrences, usually within a few years after initial surgery. ${ }^{47,48,52}$ Anti-estrogenic therapy is likely ineffective in controlling tumor growth given the lack of ER and PR immunopositivity in the high-grade component. Furthermore, although experience is limited, adjuvant therapy may provide survival benefit.

\section{Distinction from LGESS}

Clinically, patients with HGESS are more likely to present at advanced stage with extension beyond the uterus than those with LGESS. On gross examination, both can show a nodular permeative growth within the myometrium and extension into vascular spaces; however, HGESS can show destructive myometrial invasion, which is not a feature typically seen in LGESS. High-grade ESS often contains both morphologically low- and high-grade areas, but it is the presence of the latter that allows the diagnosis to be made on morphologic grounds. The high-grade areas are composed of a uniform, but distinctly larger population of epithelioid cells with round to oval vesicular nuclei arranged in a nested or corded pattern. The vascular pattern is different in these areas, being delicate and arborized as opposed to spiral arteriole-like. Mitotic activity in the epithelioid areas can be brisk, and coagulative necrosis, which is not a typical feature of LGESS, can be seen. Immunoperoxidase stains are helpful in the distinction; however, care must be taken to correlate the staining pattern with the morphologic appearance. The high-grade epithelioid areas, in contrast to LGESS (and the low-grade areas of HGESS), are typically negative for CD 10, ER, and $\mathrm{PR}$, but strongly and diffusely positive for cyclin D1. In some cases, correlation with molecular testing may be useful in this distinction as HGESS is characterized by t(10;17), whereas most LGESS have abnormalities involving 7p15 and 6p21.

\section{Distinction from Epithelioid Leiomyosarcoma}

Both HGESS and epithelioid leiomyosarcoma (eLMS) can show destructive myometrial invasion, have a tan to yellow cut surface with necrosis, and present at high stage. Moreover, both have an epithelioid component; however, eLMS typically lacks prominent delicate vasculature and the tumor cells have abundant granular to eosinophilic cytoplasm. HGESS may also have a low-grade appearing component that resembles the fibrous or fibromyxoid variant of LGESS. In contrast, if an eLMS is mixed with a spindled component, this spindled area has the appearance of a conventional LMS. In addition, eLMS is typically positive for desmin, caldesmon, ER, and PR, but negative (or only focally positive) for cyclin D1.

\section{Distinction of HGESS from Gastrointestinal Stromal Sarcoma}

As patients with HGESS often suffer recurrent disease or present at high stage (disease outside the uterus), it is important to be aware of their distinction from other peritoneal spindle cell tumors, the most common pitfall based on morphologic overlap being gastrointestinal stromal tumor (GIST). GISTs also can have a combination of spindle and epithelioid areas although they are usually more intimately admixed than in high-grade ESS. Moreover, the spindle cells of GIST typically have more brightly eosinophilic cytoplasm. One important pitfall to keep in mind is that HGESS can be diffusely positive for c-kit; however, HGESS lacks DOG1 expression and kit mutations as typically seen in GIST. ${ }^{51}$

\section{Distinction of HGESS from Undifferentiated Uterine Carcinoma}

Undifferentiated uterine carcinoma, which may occur in pure form or in combination with a lowgrade endometrioid adenocarcinoma ('de-differentiated endometrioid adenocarcinoma') is composed of a diffuse proliferation of epithelioid cells, and may, particularly in biopsy/curettage specimens with limited material, fall into the differential diagnosis of a HGESS. Further compounding the difficulty in this distinction is the finding that undifferentiated carcinoma is often diffusely positive for cyclin D1. ${ }^{53}$ Features helpful in this distinction are finding focal positivity for EMA, broad spectrum cytokeratin or CD10 in the latter. In cases in which positivity for these markers is lacking, molecular studies might be necessary for the diagnosis.

\section{Practical take-home points}

(1) Mitotic rate should not be used as the sole criterion in the distinction of LGESS from HGESS. Although most LGESS have a low mitotic rate (usually $<5$ per 10 high power fields), it can vary widely. However, in a tumor with marked mitotic activity (eg, >20-30 mitoses/10 HPF), the possibility of an HGESS should at least be considered if morphologic features are unusual. If after extensive sampling the tumor has the typical morphologic appearance of an LGESS, then it should be classified as such regardless of the mitotic rate.

(2) Distinction of high- from low-grade ESS is clinically relevant as patients with the former 
have a more aggressive clinical course. Moreover, the high-grade component lacks hormone receptors, and thus patients do not respond to hormonal treatment.

(3) In an ESS that has a fibrous or myxoid appearance, additional sampling may be prudent to exclude a high-grade component, particularly if the tumor is high stage.

(4) Epithelioid LMS is typically positive for smooth muscle and hormone receptor markers allowing distinction from HGESS.

(5) As HGESS can be diffusely positive for c-kit; this marker is not useful in the differential diagnosis of a spindle and epithelioid tumor involving the peritoneum. DOG1 is more specific in the distinction between HGESS and GIST.

(6) Both undifferentiated uterine carcinoma and high-grade ESS can be diffusely positive for cyclin D1; however, the former usually shows focal positivity for EMA and/or broad spectrum cytokeratin.

\section{Undifferentiated Uterine Sarcoma}

UUS is a high-grade sarcoma that lacks a specific line of differentiation. This is a diagnosis of exclusion and from a practical point, other uterine malignancies must be ruled out (most often achieved with extensive sampling). This is a rare tumor that typically occurs in postmenopausal women. Patients usually present with abnormal uterine bleeding, an enlarged uterus/pelvic mass, and/or symptoms related to extra-uterine spread. On gross examination, it is typically poorly circumscribed and forms an intramural or polypoid intracavitary, tan to white mass with a fleshy cut surface; necrosis/hemorrhage is common. Histologically, UUS has been subdivided into two histologic appearances-uniform (UUS-u) and pleomorphic (UUS-p) by Kurihara et al. ${ }^{54}$ It is important to note that, as described, UUS-u not only shows morphologic and immunophenotypic overlap with high-grade ESS, but also most tumors classified as such have subsequently been shown to harbor $t$ $(10 ; 17)$ and thus for purposes of classification, many but not all, are now recognized as high-grade ESS. ${ }^{55}$ UUS-p typically shows destructive myometrial infiltration, a fascicular or patternless growth pattern and is composed of highly pleomorphic cells with no resemblance to proliferative phase endometrial stroma. These tumors are typically CD10, p53, and cyclin D1 positive (which can be diffuse), but are usually negative for ER and PR. As previously stated, although many UUS-u represent high-grade ESS (morphologically, immunophenotypically, and genetically), there are UUS-u that are positive for CD10 and lack the defining genetic rearrangement of high-grade ESS; it remains to be seen as to whether these tumors represent a variant of high-grade ESS with a different, but related, genetic abnormality or represent dedifferentiation of a low-grade ESS. ${ }^{48}$
The prognosis of UUS-p is dismal with a mean survival of less than 2 years.

\section{Practical Take-Home Points}

(1) UUS is a diagnosis of exclusion and should only be made after extensive sampling at the time of hysterectomy.

(2) Most UUS are polypoid and intracavitary and sampling of the edge of the mass or the adjacent endometrium may disclose areas diagnostic of carcinosarcoma or adenosarcoma.

(3) The finding of CD10 positivity in a UUS-u excludes high-grade ESS as it is currently defined, even if cyclin D1 is diffusely positive; however, whether these tumors represent a yet to be genetically defined subset of high-grade stromal neoplasia remains to be seen.

\section{Disclosure/conflict of interest}

The author declares no conflict of interest.

\section{References}

1 Conklin CM, Longacre TA. Endometrial stromal tumors: the new WHO classification. Adv Anat Pathol 2014;21:383-393.

2 Lee CH, Nucci MR. Endometrial stromal sarcoma-the new genetic paradigm. Histopathology 2015;67:1-19.

3 Ali RH, Rouzbahman M. Endometrial stromal tumours revisited: an update based on the 2014 WHO classification. J Clin Pathol 2015;68:325-332.

4 Dionigi A, Oliva E, Clement PB et al. Endometrial stromal nodules and endometrial stromal tumors with limited infiltration: a clinicopathologic study of 50 cases. Am J Surg Pathol 2002;26:567-581.

5 Tavassoli FA, Norris HJ. Mesenchymal tumours of the uterus. VII. A clinicopathological study of 60 endometrial stromal nodules. Histopathology 1981;5:1-10.

6 Oliva E, Clement PB, Young RH et al. Mixed endometrial stromal and smooth muscle tumors of the uterus: A clinicopathologic study of 15 cases. Am J Surg Pathol 1998;22:997-1005.

7 Lloreta J, Prat J. Endometrial stromal nodule with smooth and skeletal muscle components simulating stromal sarcoma. Int J Gynecol Pathol 1992;11:293-298.

8 McCluggage WG, Sumathi VP, Maxwell P. CD10 is a sensitive and diagnostically useful immunohistochemical marker of normal endometrial stroma and of endometrial stromal neoplasms. Histopathology 2001;39:273-278.

9 Huang HY, Ladanyi M, Soslow RA. Molecular detection of JAZF1-JJAZ1 gene fusion in endometrial stromal neoplasms with classic and variant histology: evidence for genetic heterogeneity. Am J Surg Pathol 2004;28: 224-232.

10 Koontz JI, Soreng AL, Nucci M et al. Frequent fusion of the JAZF1 and JJAZ1 genes in endometrial stromal tumors. Proc Natl Acad Sci USA 2001;98:6348-6353.

11 Nucci MR, Harburger D, Koontz J et al. Molecular analysis of the JAZF1-JJAZ1 gene fusion by RT-PCR 
and fluorescence in situ hybridization in endometrial stromal neoplasms. Am J Surg Pathol 2007;31:65-70.

12 Oliva E, de Leval L, Soslow RA et al. High frequency of JAZF1-JJAZ1 gene fusion in endometrial stromal tumors with smooth muscle differentiation by interphase FISH detection. Am J Surg Pathol 2007;31: 1277-1284.

13 Stemme S, Ghaderi M, Carlson JW. Diagnosis of endometrial stromal tumors: a clinicopathologic study of 25 biopsy specimens with identification of problematic areas. Am J Clin Pathol 2014;141:133-139.

14 Oliva E, Young RH, Clement PB et al. Cellular benign mesenchymal tumors of the uterus. A comparative morphologic and immunohistochemical analysis of 33 highly cellular leiomyomas and six endometrial stromal nodules, two frequently confused tumors. Am J Surg Pathol 1995;19:757-768.

15 Chang KL, Crabtree GS, Lim-Tan SK et al. Primary uterine endometrial stromal neoplasms. A clinicopathologic study of 117 cases. Am J Surg Pathol 1990; 14:415-438.

16 Evans HL. Endometrial stromal sarcoma and poorly differentiated endometrial sarcoma. Cancer 1982;50: 2170-2182.

17 Fekete PS, Vellios F. The clinical and histologic spectrum of endometrial stromal neoplasms: a report of 41 cases. Int J Gynecol Pathol 1984;3:198-212.

18 Oliva E, Clement PB, Young RH. Endometrial stromal tumors: an update on a group of tumors with a protean phenotype. Adv Anat Pathol 2000;7:257-281.

19 Clement PB. The pathology of uterine smooth muscle tumors and mixed endometrial stromal-smooth muscle tumors: a selective review with emphasis on recent advances. Int J Gynecol Pathol 2000;19:39-55.

20 Clement PB, Scully RE. Endometrial stromal sarcomas of the uterus with extensive endometrioid glandular differentiation: a report of three cases that caused problems in differential diagnosis. Int J Gynecol Pathol 1992;11:163-173.

21 Oliva E, Young RH, Clement PB et al. Myxoid and fibrous endometrial stromal tumors of the uterus: a report of 10 cases. Int J Gynecol Pathol 1999;18:310-319.

22 Oliva E, Clement PB, Young RH. Epithelioid endometrial and endometrioid stromal tumors: a report of four cases emphasizing their distinction from epithelioid smooth muscle tumors and other oxyphilic uterine and extrauterine tumors. Int J Gynecol Pathol 2002;21: $48-55$.

23 Yilmaz A, Rush DS, Soslow RA. Endometrial stromal sarcomas with unusual histologic features: a report of 24 primary and metastatic tumors emphasizing fibroblastic and smooth muscle differentiation. Am J Surg Pathol 2002;26:1142-1150.

24 Parra-Herran CE, Yuan L, Nucci MR et al. Targeted development of specific biomarkers of endometrial stromal cell differentiation using bioinformatics: The IFITM1 model. Mod Pathol 2014;27:569-579.

25 Oliva E, Young RH, Amin MB et al. An immunohistochemical analysis of endometrial stromal and smooth muscle tumors of the uterus: a study of 54 cases emphasizing the importance of using a panel because of overlap in immunoreactivity for individual antibodies. Am J Surg Pathol 2002;26:403-412.

26 Baker RJ, Hildebrandt RH, Rouse RV et al. Inhibin and CD99 (MIC2) expression in uterine stromal neoplasms with sex-cord-like elements. Hum Pathol 1999;30: 671-679.
27 Nucci MR, O’Connell JT, Huettner PC et al. h-caldesmon expression effectively distinguishes endometrial stromal tumors from uterine smooth muscle tumors. Am J Surg Pathol 2001;25:455-463.

28 Rush DS, Tan J, Baergen RN et al. h-caldesmon, a novel smooth muscle-specific antibody, distinguishes between cellular leiomyoma and endometrial stromal sarcoma. Am J Surg Pathol 2001;25:253-258.

29 Albores-Saavedra J, Dorantes-Heredia R, Chable-Montero $\mathrm{F}$ et al. Endometrial stromal sarcomas: immunoprofile with emphasis on HMB45 reactivity. Am J Clin Pathol 2014;141:850-855.

30 Dal Cin P, Aly MS, De Wever I et al. Endometrial stromal sarcoma $\mathrm{t}(7 ; 17)(\mathrm{p} 15-21 ; \mathrm{q} 12-21)$ is a nonrandom chromosome change. Cancer Genet Cytogenet 1992;63: 43-46.

31 Micci F, Walter CU, Teixeira MR et al. Cytogenetic and molecular genetic analyses of endometrial stromal sarcoma: nonrandom involvement of chromosome arms $6 p$ and $7 p$ and confirmation of JAZF1/JJAZ1 gene fusion in t(7;17). Cancer Genet Cytogenet 2003;144:119-124.

32 Chiang S, Oliva E. Recent developments in uterine mesenchymal neoplasms. Histopathology 2013;62: 124-137.

33 Micci F, Panagopoulos I, Bjerkehagen B et al. Consistent rearrangement of chromosomal band 6p21 with generation of fusion genes JAZF1/PHF1 and EPC1/ PHF1 in endometrial stromal sarcoma. Cancer Res 2006;66:107-112.

34 D’Angelo E, Ali RH, Espinosa I et al. Endometrial stromal sarcomas with sex cord differentiation are associated with PHF1 rearrangement. Am J Surg Pathol 2013;37:514-521.

35 Dewaele B, Przybyl J, Quattrone A et al. Identification of a novel, recurrent MBTD1-CXorf67 fusion in lowgrade endometrial stromal sarcoma. Int J Cancer 2014;134:1112-1122.

36 Panagopoulos I, Thorsen J, Gorunova L et al. Fusion of the ZC3H7B and BCOR genes in endometrial stromal sarcomas carrying an X;22-translocation. Genes Chromosomes Cancer 2013;52:610-618.

37 Schoolmeester JK, Sciallis AP, Greipp PT et al. Analysis of MDM2 amplification in 43 endometrial stromal tumors: a potential diagnostic pitfall. Int J Gynecol Pathol 2015;34:576-583.

38 Chew I, Oliva E. Endometrial stromal sarcomas: a review of potential prognostic factors. Adv Anat Pathol 2010;17:113-121.

39 Rauh-Hain JA, Goodman A, Boruta DM et al. Endometrial stromal sarcoma: a clinicopathologic study of 29 patients. J Reprod Med 2014;59:547-552.

40 Clement PB. Intravenous leiomyomatosis of the uterus. Pathol Annu 1988;23(Pt 2):153-183.

41 Clement PB, Young RH, Scully RE. Intravenous leiomyomatosis of the uterus. A clinicopathological analysis of 16 cases with unusual histologic features. Am J Surg Pathol 1988;12:932-945.

42 Mulvany NJ, Slavin JL, Ostor AG et al. Intravenous leiomyomatosis of the uterus: a clinicopathologic study of 22 cases. Int J Gynecol Pathol 1994;13:1-9.

43 Clement PB, Scully RE. Uterine tumors resembling ovarian sex-cord tumors. A clinicopathologic analysis of fourteen cases. Am J Clin Pathol 1976;66:512-525.

44 Irving JA, Carinelli S, Prat J. Uterine tumors resembling ovarian sex cord tumors are polyphenotypic neoplasms with true sex cord differentiation. Mod Pathol 2006;19: 17-24. 
45 Krishnamurthy S, Jungbluth AA, Busam KJ et al. Uterine tumors resembling ovarian sex-cord tumors have an immunophenotype consistent with true sex-cord differentiation. Am J Surg Pathol 1998;22:1078-1082.

46 Lee CH, Ou WB, Marino-Enriquez A et al. 14-3-3 fusion oncogenes in high-grade endometrial stromal sarcoma. Proc Natl Acad Sci USA 2012;109:929-934.

47 Lee CH, Marino-Enriquez A, Ou W et al. The clinicopathologic features of YWHAE-FAM22 endometrial stromal sarcomas: a histologically high-grade and clinically aggressive tumor. Am J Surg Pathol 2012;36: 641-653.

48 Sciallis AP, Bedroske PP, Schoolmeester JK et al. Highgrade endometrial stromal sarcomas: a clinicopathologic study of a group of tumors with heterogenous morphologic and genetic features. Am J Surg Pathol 2014;38:1161-1172.

49 Croce S, Hostein I, Ribeiro A et al. YWHAE rearrangement identified by FISH and RT-PCR in endometrial stromal sarcomas: genetic and pathological correlations. Mod Pathol 2013;26:1390-1400.

50 Lee CH, Ali RH, Rouzbahman M et al. Cyclin D1 as a diagnostic immunomarker for endometrial stromal sarcoma with YWHAE-FAM22 rearrangement. Am J Surg Pathol 2012;36:1562-1570.

51 Lee CH, Hoang LN, Yip S et al. Frequent expression of KIT in endometrial stromal sarcoma with YWHAE genetic rearrangement. Mod Pathol 2014;27:751-757.

52 Kruse AJ, Croce S, Kruitwagen RF et al. Aggressive behavior and poor prognosis of endometrial stromal sarcomas with YWHAE-FAM22 rearrangement indicate the clinical importance to recognize this subset. Int J Gynecol Cancer 2014;24:1616-1622.

53 Shah VI, McCluggage WG. Cyclin D1 does not distinguish YWHAE-NUTM2 high-grade endometrial stromal sarcoma from undifferentiated endometrial carcinoma. Am J Surg Pathol 2015;39:722-724.

54 Kurihara S, Oda Y, Ohishi Y et al. Endometrial stromal sarcomas and related high-grade sarcomas: immunohistochemical and molecular genetic study of 31 cases. Am J Surg Pathol 2008;32:1228-1238.

55 Gremel G, Liew M, Hamzei $\mathrm{F}$ et al. A prognosis based classification of undifferentiated uterine sarcomas: identification of mitotic index, hormone receptors and YWHAE-FAM22 translocation status as predictors of survival. Int J Cancer 2015;136:1608-1618. 